\title{
Hadronic interactions of primary cosmic rays with the FLUKA code
}

\author{
M. N. Mazziotta* \\ Istituto Nazionale di Fisica Nucleare, Sezione di Bari, 70126 Bari, Italy \\ E-mail: mazziotta@ba.infn.it \\ F. Cerutti \\ CERN, Geneva, Switzerland \\ E-mail: Francesco.Cerutti@cern.ch
}

\author{
A. Ferrari \\ CERN, Geneva, Switzerland \\ E-mail: Alfredo.Ferrari@cern.ch \\ D. Gaggero
}

SISSA, via Bonomea 265, 34136 Trieste, Italy

INFN, Sezione di Trieste, via Valerio 2, 34127 Trieste, Italy

E-mail: Daniele.Gaggeroesissa.it

\section{F. Loparco}

Dipartimento di Fisica "M. Merlin" dell'Università e del Politecnico di Bari, I-70126 Bari, Italy Istituto Nazionale di Fisica Nucleare, Sezione di Bari, 70126 Bari, Italy

E-mail: Francesco.Loparco@ba.infn.it

\section{P. R. Sala}

Istituto Nazionale di Fisica Nucleare, Sezione di Milano, 20133 Milano, Italy

E-mail: Paola.Sala@mi.infn.it

\begin{abstract}
The measured fluxes of secondary particles produced by the interactions of cosmic rays with the astronomical environment represent a powerful tool to infer some properties of primary cosmic rays. In this work we investigate the production of secondary particles in inelastic hadronic interactions between several cosmic rays species of projectiles and different target nuclei of the interstellar medium. The yields of secondary particles have been calculated with the FLUKA simulation package, that provides with very good accuracy the energy distributions of secondary products in a large energy range. An application to the propagation and production of secondaries in the Galaxy is presented.
\end{abstract}

The 34th International Cosmic Ray Conference,

30 July- 6 August, 2015

The Hague, The Netherlands

\footnotetext{
* Speaker.
} 


\section{Introduction}

According to the currently accepted scenario, Galactic Cosmic Rays (CRs) are accelerated in Supernova Remnants (SNRs) and interact with the turbulent interstellar magnetic field: the resulting motion is well described by a diffusion equation. During their journey through the Galaxy, the inelastic collisions of hadronic CRs with the interstellar medium (ISM), usually dubbed spallation processes, produce lighter particles and secondary radiation: the study of these events is very important since it may shed light on the origin of the CRs themselves and on the mechanisms governing their transport.

The production of light nuclei - such as Boron - from heavier ones (in particular Carbon and Nitrogen) has been extensively studied in recent times, since light nuclei ratios (e.g. B/C, N/O) are often used to constrain the propagation models in the Galaxy and in particular the rigidity dependence and normalization of the diffusion coefficient.

The collisions of protons and Helium nuclei with the gas, and subsequent decays of the produced neutral pions $\left(\pi^{0} \mathrm{~s}\right)$, are expected to give the most relevant contribution to the gammaray diffuse emission in the Galactic plane, since that is the region with the largest gas column densities. In this context, the high precision gamma-ray maps and spectra measured by the FermiLAT instrument in the energy band spanning from several tens of $\mathrm{MeV}$ to several hundreds of $\mathrm{GeV}$ are very useful and represent a unique opportunity to understand the CR transport properties in different regions of the Galaxy [1].

Charged leptons and antiprotons are another important product of the inelastic hadronic collisions of CRs with the interstellar gas. The PAMELA collaboration measured the positron, electron and antiproton spectra, as well as the spectra of many light nuclei [2]. Recently the AMS02 collaboration provided very accurate results on the positron fraction and new results on the positron, electron and proton intensities, as well as on the helium spectrum and on the $\mathrm{B} / \mathrm{C}$ ratio [3]. All those datasets were taken during the same period, and this circumstance makes the interpretation of the data easier. Simultaneous measurements of several particle spectra performed by the same experiment over a wide energy range will in fact ensure reduced experimental systematics and will also limit the uncertainties arising from the CR propagation in the heliosphere (solar modulation) and in the Galaxy.

A common practice in the literature is to fit different observables (e.g. hadronic and leptonic spectra) using cross sections obtained from several parameterizations, derived with different methods and under different assumpions: this may lead to inconsistencies in the determination of CR diffusion models. In the present work we aim at providing a complete and consistent set of cross sections for the secondary production of hadrons and leptons. We perform our study with the FLUKA MC simulation code [4], and we present a comprehensive calculation of the secondary hadron, lepton, gamma-ray and neutrino yields produced by the inelastic interactions between several species of stable or long-lived cosmic rays projectiles (p, D, T, ${ }^{3} \mathrm{He},{ }^{4} \mathrm{He},{ }^{6} \mathrm{Li},{ }^{7} \mathrm{Li},{ }^{9} \mathrm{Be}$, ${ }^{10} \mathrm{Be},{ }^{10} \mathrm{~B},{ }^{11} \mathrm{~B},{ }^{12} \mathrm{C},{ }^{13} \mathrm{C},{ }^{14} \mathrm{C},{ }^{14} \mathrm{~N},{ }^{15} \mathrm{~N},{ }^{16} \mathrm{O},{ }^{17} \mathrm{O},{ }^{18} \mathrm{O},{ }^{20} \mathrm{Ne},{ }^{24} \mathrm{Mg}$ and ${ }^{28} \mathrm{Si}$ ) and different target gas nuclei $\left(\mathrm{p},{ }^{4} \mathrm{He},{ }^{12} \mathrm{C},{ }^{14} \mathrm{~N},{ }^{16} \mathrm{O},{ }^{20} \mathrm{Ne},{ }^{24} \mathrm{Mg}\right.$ and $\left.{ }^{28} \mathrm{Si}\right)$.

The present results provide, for the first time, a complete and self-consistent set of all the relevant inclusive cross sections regarding the whole spectrum of secondary products in nuclear collisions. We cover, for the projectiles, a kinetic energy range extending from $0.1 \mathrm{GeV} / \mathrm{n}$ up 
to $100 \mathrm{TeV} / \mathrm{n}$ in the lab frame [5]. In order to show the importance of our results for multimessenger studies about the physics of CR propagation, we evaluate the propagated spectra of Galactic secondary nuclei, leptons, and gamma rays produced by the interactions of CRs with the insterstellar gas, exploiting the numerical code DRAGON [6].

\section{Secondary particle production in CR interactions with FLUKA}

FLUKA is a general purpose Monte Carlo (MC) code for the simulation of hadronic and electromagnetic interactions. It is used in many applications, and is continuously checked using the available data from low energy nuclear physics, high-energy accelerator experiments and measurements of particle fluxes in the atmosphere. Hadronic interactions are treated in FLUKA following a theory-driven approach [7]. The general phenomenology is obtained from a microscopical description of the interactions between the fundamental constituents (quarks and nucleons), appropriate for the different energy regions. Below a few $\mathrm{GeV}$, the hadron-nucleon interaction model is based on resonance production and decay of particles, while for higher energies the Dual Parton Model (DPM) is used. The extension from hadron-nucleon to hadron-nucleus interactions is done in the framework of the PreEquilibrium Approach to NUclear Thermalization model (PEANUT) [8], including the Gribov-Glauber multi-collision mechanism followed by the pre-equilibrium stage and possible equilibrium processes (evaporation, fission, Fermi break-up and gamma deexcitation).
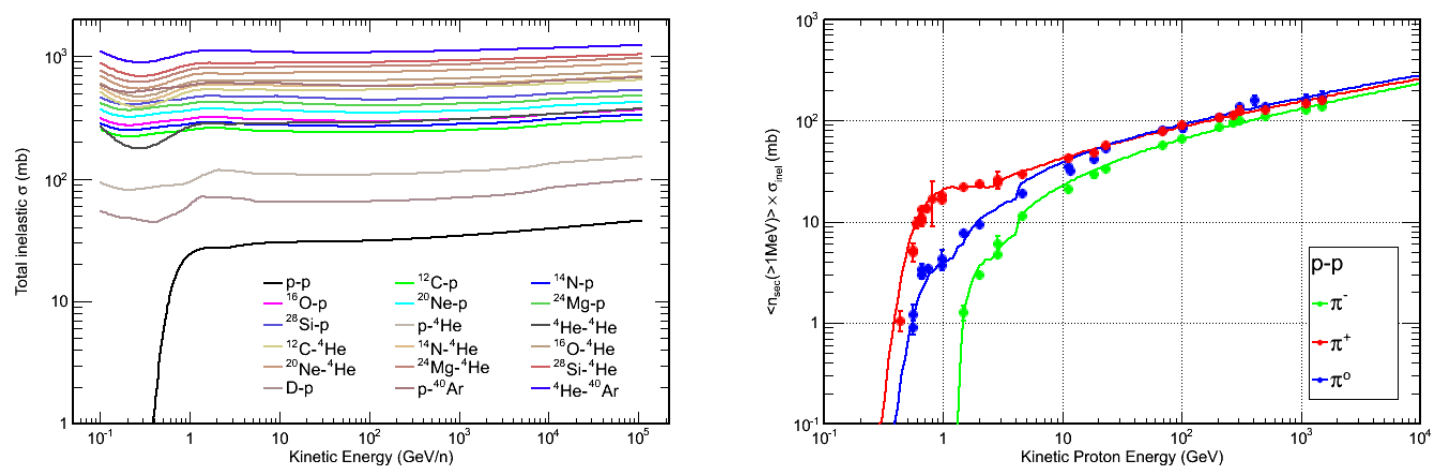

Figure 1: Left panel: Total inelastic cross sections as a function of the energy per nucleon of the incoming projectile. The plot shows the cross sections for all the projectile-target pairs studied in the present work. Right panel: Inclusive cross sections for the production of $\pi^{0}$ (blue), $\pi^{+}$(red) and $\pi^{-}$(green) in p-p collision as function of the incoming proton kinetic energy. Lines: FLUKA simulation; points: data from Ref. [9].

The first quantities we want to investigate are the total inelastic cross sections for the collisions we are focusing on, and - as an important cross check - the pion production cross section (for the proton-proton case) compared to current data. The left panel of Fig. 1 shows the total inelastic cross section calculated in the FLUKA code for the collision processes investigated in the present work. The right panel of the Fig. 1 shows the pion inclusive cross sections in p-p collisions calculated from the FLUKA simulation at the interaction level compared to current data.

With these results in hand, we can present the outcome of our analysis for all stable particles (including radionuclides whose decay is not taken into account at this stage). In Fig. 2 we show the 

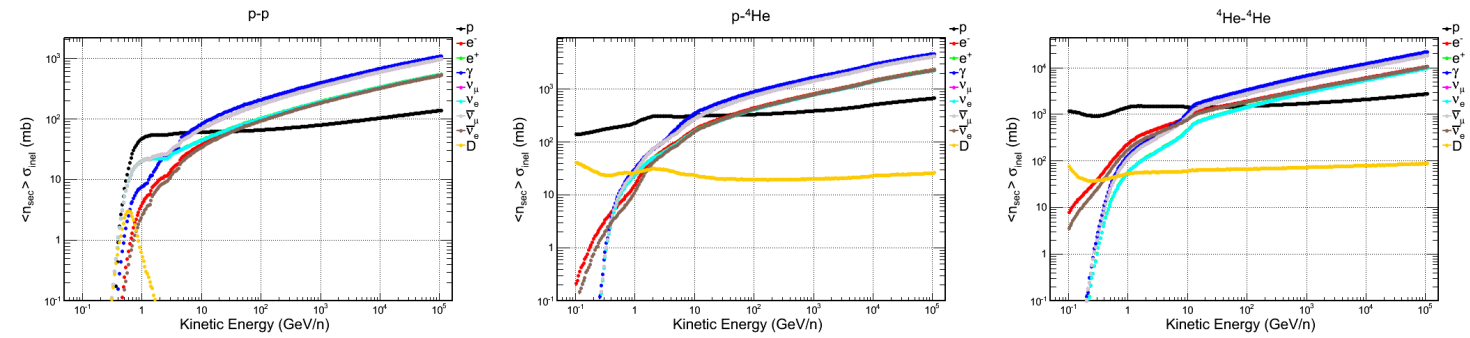

Figure 2: Inclusive cross sections for the production of protons (black), electrons (red), positrons (green), gamma rays (blue), electron neutrinos (cyan), electron antineutrinos (grey), muon neutrinos (magenta), muon antineutrinos (brown) and Deuterons (orange) in the p-p, p- ${ }^{4} \mathrm{He}$ and ${ }^{4} \mathrm{He}-{ }^{4} \mathrm{He}$ collisions.
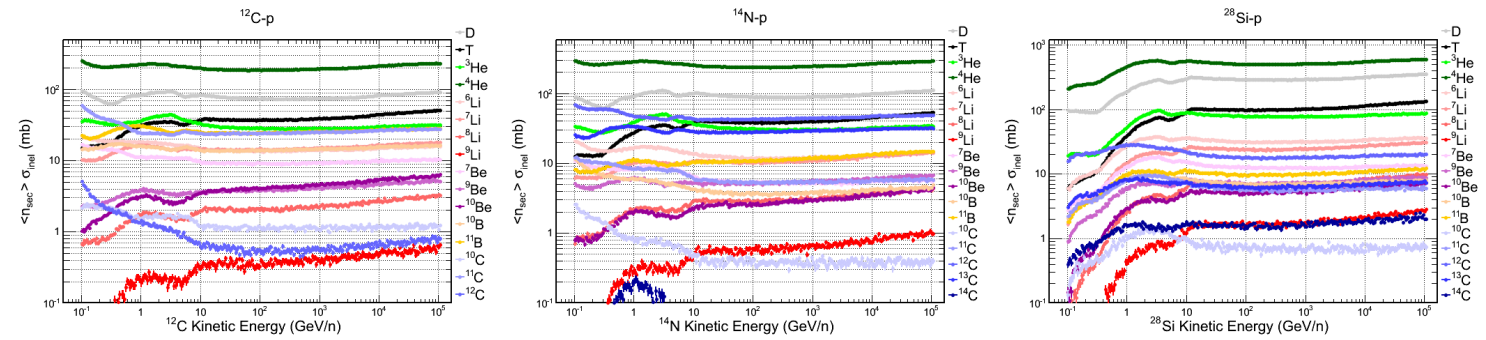

Figure 3: Inclusive cross sections for the production of spallation nuclei in collisions of ${ }^{12} \mathrm{C},{ }^{14} \mathrm{~N}$ and ${ }^{28} \mathrm{Si}$ with p. The plots show the cross sections for the production of Deuteron (gray markers), Triton (black markers) and the isotopes of $\mathrm{He}\left({ }^{3} \mathrm{He}\right.$ and ${ }^{4} \mathrm{He}$, green markers), $\mathrm{Li}\left({ }^{6} \mathrm{Li},{ }^{7} \mathrm{Li},{ }^{8} \mathrm{Li}\right.$ and ${ }^{9} \mathrm{Li}$, red markers $), \mathrm{Be}$ $\left({ }^{7} \mathrm{Be}\right.$ and ${ }^{9} \mathrm{Be}$, magenta markers), $\mathrm{B}\left({ }^{10} \mathrm{~B}\right.$ and ${ }^{11} \mathrm{~B}$, orange markers $)$ and $\mathrm{C}\left({ }^{10} \mathrm{C},{ }^{11} \mathrm{C},{ }^{12} \mathrm{C},{ }^{13} \mathrm{C}\right.$ and ${ }^{14} \mathrm{C}$, blue markers). Lighter (darker) color shades correspond to lighter (heavier) isotopes.

total inclusive cross sections for the production of protons, electrons and positrons, gamma rays, electron neutrinos and antineutrinos, muon neutrinos and antineutrinos and deuterons, as a function of the kinetic energy per nucleon of the projectile in the p-p, p- ${ }^{4} \mathrm{He}$ and ${ }^{4} \mathrm{He}-{ }^{4} \mathrm{He}$ collisions. In Fig. 3 we show some relevant results among all the calculations we performed for the heavy nuclei. We plot the inclusive cross sections of to the the interactions of ${ }^{12} \mathrm{C},{ }^{14} \mathrm{~N}$ and ${ }^{28} \mathrm{Si}$ with protons, yielding deuterons, tritons and all the isotopes of $\mathrm{He}, \mathrm{Li}, \mathrm{Be}, \mathrm{B}$ and $\mathrm{C}$.

We remark that this is the first time in which such a complete sample of nuclear cross sections are computed in a consistent way with a single numerical code. Due to the completeness and accuracy of these computations, we point out that the dataset we produced can be used by the community working on CR physics to constrain the properties of CR transport in a more solid way, as we will show in the next Section.

\section{Application to the Galactic emission}

In order to evaluate the emission from the Galaxy we use a customized version of the propagation code DRAGON (2D version) ${ }^{1}$ in which we implemented the present parameterizations in the secondary production routine.

\footnotetext{
${ }^{1}$ A version of DRAGON code is available for download at the link http://www.dragonproject.org/.
} 
We study the spectra of the following species: protons, Helium, Boron, Carbon, electrons, and positrons; for each one we evaluate the contribution of secondaries originating from nuclear interactions. In our simulation we assume that the ISM is composed of Hydrogen and Helium with relative abundances $1: 0.1$. The $\beta^{ \pm}$decays and the electron captures of unstable isotopes are taken into account according to their lifetimes. In the current version of the DRAGON code the daughter nucleus carries out all the energy of the parent nucleus, while the other products (i.e. leptons) are discarded.

The purpose of this section is to discuss the implications of the present parameterizations on some reference diffusion models. The setup we are using is standard: CR transport properties are homogeneous and isotropic; the scalar diffusion coefficient depends on the particle rigidity $R$ and on the distance from the Galactic Plane $z$ according to the following parameterization: $D=D_{0} \beta^{\eta}\left(\frac{R}{R_{0}}\right)^{\delta} e^{|z| / z_{t}}$ where $D_{0}$ is the diffusion coefficient normalization at the reference rigidity $R_{0}=4 \mathrm{GV} ; \eta$ effectively describes the complicated physical effects that may play a major role at low energy (below $1 \mathrm{GeV}$ ), e.g. the dissipation of Alfvén waves due to the resonant interaction with CRs (here $\beta$ represents as usual the particle velocity in units of the speed of light); $\delta$ is the spectral index: it is constrained by the data on the light nuclei ratios, in particular by the measurements of the $\mathrm{B} / \mathrm{C}$ ratio; $z_{t}$ is the scale height of the diffusive halo of the Galaxy.

Our starting point consists of two diffusion models considered as a reference in several previous works (see e.g. [10]) labelled as KRA $(\delta=0.5)$ and $\mathrm{KOL}(\delta=0.33)$ : they are mainly tuned on the PAMELA B/C data. We also present a new model tuned on the recent preliminary AMS02 B/C data with $\delta=0.42$ based on the one presented in [11].

In all cases the injected spectra up to Silicon nuclei are described by a broken power law with a single break at $10 \mathrm{GeV}$. In all models the spectral indices of $\mathrm{p}, \mathrm{He}$ and nuclei below the break are 2.05, 2.18 and 2.20 respectively. In the KOL/KRA/AMS models the indices above the break of $\mathrm{p}$, He and nuclei are 2.48/2.33/2.43, 2.40/2.24/2.32 and 2.60/2.40/2.50 respectively. The source term distribution is taken from [12]. The gas density distribution is taken from the public Galprop version $[13,14,15]$. We also consider a primary electron+positron extra component with harder injection spectrum in order to explain the anomalous rise of the positron fraction above $10 \mathrm{GeV}$ reported by PAMELA, Fermi-LAT and AMS02. This behaviour cannot be reproduced in the standard scenario in which positrons only originate from spallation of protons, helium and heavier nuclei on interstellar gas.

In order to compare our computations with the data, we have to consider that, in the final stage of their propagation, charged particles enter the sphere of influence of the Sun. Here they diffuse in the Heliospheric magnetic field, and suffer adiabatic energy losses and convection due to the presence of the solar wind: this process is called solar modulation and is relevant for low energy $(<10 \mathrm{GeV})$ particles. In this work we adopt the so-called "force field approximation" [16], a simplified description in which a single free parameter is involved: the modulation potential $\phi$.

Given all these ingredients, our method is the following: we compare the current data to the computations performed with our modified version of DRAGON with the new cross section parameterizations and we retune the model accordingly. Our results are summarized in Fig. 4. Very remarkably, we find that it is possible to reproduce correctly all the relevant observables (namely, proton, Helium, light nuclei spectra, and B/C ratio) with the reference models considered, 

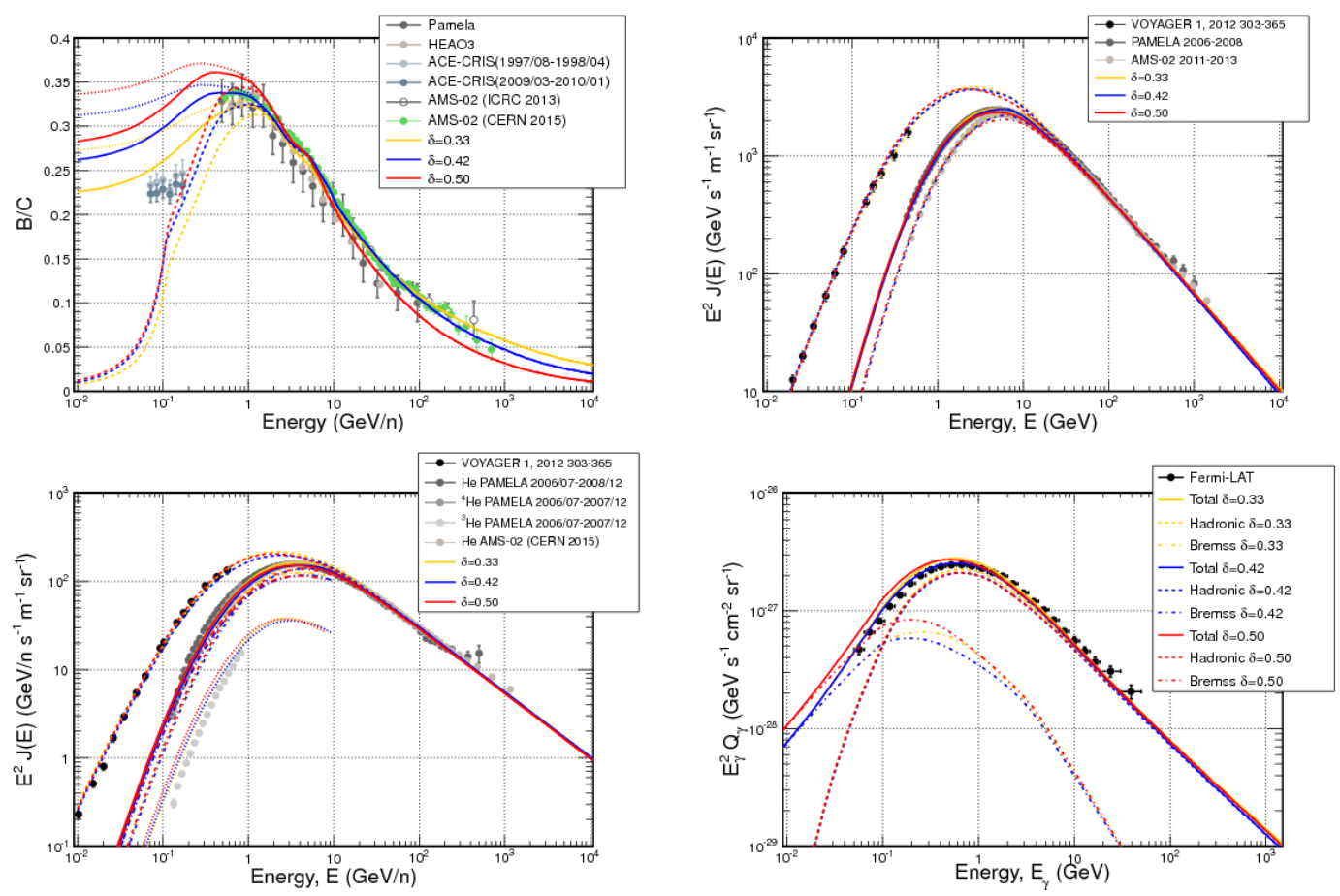

Figure 4: DRAGON results. The models are: $\delta=0.33$ (KOL), $\delta=0.42$ and $\delta=0.50$ (KRA). Top left: Boron to Carbon ratio; bottom left: Helium; top right: proton, comparison with PAMELA, AMS02 and VOYAGER1 [17] data. Dashed line: unmodulated intensity; solid (dotted) line: modulated intensity by means of the force field approximation with $\phi=0.42$ (0.62) GV (some data point are quoted from [18]). The bottom right panel shows the differential gamma-ray emissivity as a function of the gamma-ray energy, evaluated by folding the proton and the Helium (hadronic), electron (bremsstrahlung) LIS with the $\gamma$-ray production cross sections (the data points are quoted from [21]).

after a rescaling of the diffusion coefficient normalization $\left(D_{0}\right)$ and a minor fine-tuning of the other parameters. For the KOL model we use $D_{0}=4.25 \times 10^{28} \mathrm{~cm}^{2} \mathrm{~s}^{-1}, \eta=0$ and $v_{\mathrm{A}}=33 \mathrm{~km} \mathrm{~s}^{-1}$ (Alfvén velocity), while for the KRA model we use $D_{0}=2.8 \times 10^{28} \mathrm{~cm}^{2} \mathrm{~s}^{-1}, \eta=-0.4$ and $v_{\mathrm{A}}=17.5 \mathrm{~km} \mathrm{~s}^{-1}$. Finally for the AMS02 model we use $D_{0}=3.35 \times 10^{28} \mathrm{~cm}^{2} \mathrm{~s}^{-1}, \eta=-0.4$ and $v_{\mathrm{A}}=17.5 \mathrm{~km} \mathrm{~s}^{-1}$.

For the three models we use the solar modulation parameter $\phi=0.42 \mathrm{GV}$ and $0.62 \mathrm{GV}$ for all the species to reproduce the PAMELA and AMS02 data respectively. The differences of these results with respect to the PAMELA and AMSO2 proton data are within $\pm 10 \%$ or better. Clearly, the model with $\delta=0.42$ is designed to have the best compatibility with the AMS02 preliminary $\mathrm{B} / \mathrm{C}$ data. It is worth to point out that in these three models we do not consider the presence of the break around $300 \mathrm{GeV}$ in the proton and nuclei spectra, since it is beyond the aim of this paper. However, with this complete sample of inclusive cross sections we do not need to introduce the concept of nuclear enhancement factor to describe secondary production in interaction among heavier nuclei, extrapolating the results of p-p interaction [19]. In particular, this factor depends on the shape of the spectrum (see for instance [20]) and it does not work in case of abrupt change in the CR spectra (as in case of breaks). 
Here we want to stress that with this new evaluation we have a much better insight on the nuclear processes, since the cross-sections can be computed accurately in a wide energy range. For example, given the more accurate determination of the gamma-ray emissivity, we can turn our attention to the Local Interstellar Spectra (LIS) of protons and Helium nuclei, not affected by solar modulation. This quantity can be inferred from the Fermi-LAT gamma-ray data [21] and its determination is useful in order to constrain the propagation models described above, since each of them implies a different shape and normalization for the LIS. The bottom-right panel of Fig. 4 shows the gamma-ray emissivity calculated by means of the proton, Helium and electron LIS. We calculate the contribution associated with the hadronic collisions and bremsstrahlung by folding the proton, Helium and electron LIS with the corresponding $\gamma$-ray production cross sections on the ISM with relative abundances of $\mathrm{H}: \mathrm{He}: \mathrm{C}: \mathrm{N}: \mathrm{O}: \mathrm{Ne}: \mathrm{Mg}: \mathrm{Si}=$ $1: 0.096: 4.65 \times 10^{-4}: 8.3 \times 10^{-5}: 8.3 \times 10^{-4}: 1.3 \times 10^{-4}: 3.9 \times 10^{-5}: 3.69 \times 10^{-5}$. An inspection of this plot shows that at low energies the contribution from electron bremsstrahlung is not negligible. This contribution mainly depends on the electron spectrum, that should also be constrained taking synchrotron radiation into account (see for instance [22, 23]).

\section{Conclusions}

We have used the FLUKA simulation code to evaluate the inclusive cross sections for the production of stable secondary particles and as well as nuclei from spallation in the interactions of several cosmic-ray projectiles with different target nuclei.

As an example of application we implemented the values of the cross sections in a custom version of the DRAGON CR propagation code, showing that it is possible to reproduce with accuracy the measured CR spectra and light nuclei ratios as well as the gamma-ray emissivity.

\section{References}

[1] M. Ackermann et al. [Fermi-LAT Collaboration], Astrophys. J. 750 (2012) 3

[2] O. Adriani et al. [PAMELA Collaboration], Nature 458 (2009) 607

O. Adriani et al. [PAMELA Collaboration], Phys. Rev. Lett. 105 (2010) 121101

O. Adriani et al. [PAMELA Collaboration], Science 332 (2011) 69

O. Adriani et al. [PAMELA Collaboration], Phys. Rev. Lett. 106 (2011) 201101

O. Adriani et al. [PAMELA Collaboration], Phys. Rev. Lett. 111 (2013) 8, 081102

O. Adriani et al. [PAMELA Collaboration], Astrophys. J. 765, 91 (2013)

O. Adriani et al. [PAMELA Collaboration], Astrophys. J. 770 (2013) 2

[3] M. Aguilar et al. [AMS Collaboration], Phys. Rev. Lett. 110 (2013) 14, 141102.

L. Accardo et al. [AMS Collaboration], Phys. Rev. Lett. 113, 121101 (2014).

M. Aguilar et al. [AMS Collaboration], Phys. Rev. Lett. 113 (2014) 121102.

M. Aguilar [AMS Collaboration], Phys. Rev. Lett. 114, no. 17, 171103 (2015).

http://www.ams02.org/2013/07/new-results-from-ams-presented-at-icrc-2013/

https://indico.cern.ch/event/381134/other-view?view=standard\#20150415 
[4] G. Battistoni, S. Muraro, P.R. Sala, F. Cerutti, A. Ferrari, S. Roesler, A. Fassò, J. Ranft, The FLUKA code: Description and benchmarking, Proceedings of the Hadronic Shower Simulation Workshop 2006, Fermilab 6-8 September 2006, M. Albrow, R. Raja Eds., AIP Conference Proceeding 896, 31-49, (2007)

A. Ferrari, P.R. Sala, A. Fassò, and J. Ranft, FLUKA: a multi-particle transport code CERN-2005-10 (2005), INFN/TC_05/11, SLAC-R-773

http://www.fluka.org

[5] M. N. Mazziotta, F. Cerutti, A. Ferrari, D. Gaggero, F. Loparco and P. R. Sala Production of secondary particles and nuclei in cosmic rays collisions with the insterstellar gas using the FLUKA code, to be submitted

[6] C. Evoli, D. Gaggero, D. Grasso and L. Maccione, JCAP 0810 (2008) 018

D. Gaggero, L. Maccione, G. Di Bernardo, C. Evoli and D. Grasso, Phys. Rev. Lett. 111 (2013) 2, 021102

[7] A. Ferrari and P.R. Sala The Physics of High Energy Reactions, Proc. Workshop on Nuclear Reaction Data and Nuclear Reactors Physics, Design and Safety, International Centre for Theoretical Physics, Miramare-Trieste, Italy, 15 April-17 May 1996, Ed. A. Gandini and G. Reffo, World Scientific, p. 424 (1998)

[8] A. Fassò, A. Ferrari, J. Ranft, P.R. Sala, FLUKA: status and prospective for hadronic applications, in: A. Kling, F. Barão, M. Nakagawa, L. Távora, P. Vaz (Eds.) Proceedings of the Monte Carlo 2000 Conference, Lisbon, October 23-26 2000, Springer-Verlag, Berlin, 2001, p. 955-960

G. Battistoni et al. Recent developments in the FLUKA nuclear reaction models Proc. 11th Int. Conf. on Nucl. React. Mech. (Varenna, Italy, 12-16 June 2006) 483

[9] C.D. Dermer, Astrophysical Journal 307 (1986) 47

[10] C. Evoli, D. Gaggero, D. Grasso and L. Maccione, Phys. Rev. Lett. 108, 211102 (2012)

[11] C. Evoli, D. Gaggero, and D. Grasso [arXiv:1504.05175 [astro-ph.HE]]

[12] K. M. Ferriere, Rev. Mod. Phys. 73, 1031 (2001)

[13] http://galprop.stanford.edu/

[14] A.W.Strong and I.V. Moskalenko, ApJ, 509, Issue 1, pp. 212-228 (1998)

[15] I.V. Moskalenko et al., ApJ, 565, Issue 1, pp. 280-296 (2002)

[16] L.J. Gleeson and W.I. Axford, ApJ 149, L115 (1967)

L.J. Gleeson and W.I. Axford, ApJ 154, 1011 (1968)

[17] E.C. Stone et al., Science 341 (2013) 150

[18] D. Maurin, F. Melot and R. Taillet, A\&A 569 (2014) A32 [arXiv:1302.5525]

[19] M. Mori, Astroparticle Physics 31 (2009) 341

[20] M. Kachelriess, I. V. Moskalenko and S. S. Ostapchenko, Astrophys. J. 789 (2014) 136

[21] J.M. Casandjian Local HI emissivity measured with the Fermi-LAT and implications for cosmic-ray spectra, Accepted in ApJ [arXiv:1506.00047 [astro-ph.HE]]

[22] G. Di Bernardo, C. Evoli, D. Gaggero, D. Grasso and L. Maccione, JCAP 1303 (2013) 036

[23] E. Orlando and A. Strong, MNRAS 436 (2013) 2127 